\section{Clinician Corner}

\section{Catherine Watson Genna, BS, IBCLC, RLC ${ }^{a}$}

Epigenetics, the study of factors that affect gene expression without changing the DNA (deoxyribonucleic acid) sequence, is a hot new area in science. Epigenetic research hints at differences in gene expression between breastfed and artificially fed infants. DNA methylation is the most studied of the epigenetic mechanisms that control gene expression.

\section{Genetics and Epigenetics Primer}

DNA is the chemical that stores genetic information in the nucleus of each cell. The DNA in the nucleus is called the genome, and it contains the assembly instructions for the entire body, in the form of four "bases" (adenine, which pairs with thymine, and guanine which pairs with cytosine, denoted by their first initials). When genes are read, they are copied into messenger RNA (ribonucleic acid), like little note cards copied from a giant reference book. The messenger RNA bases are read three at a time to code for amino acids, the building blocks of proteins. The sequence of amino acids determines how a protein folds, and that folding produces a three-dimensional shape responsible for the protein's function.

Think of a puzzle piece that has gotten wet and become warped-it will no longer fit and will leave a hole in the picture; or think of a key that has been run over by a trainit can't open its lock. Mutated (changed) genes produce proteins that don't work properly. Sometimes a mutation will insert a "stop" signal, which prevents the protein from being made at all.

Cellular enzymes called DNA polymerases copy the DNA when cells divide. When certain proteins are needed for cell maturation or metabolism, RNA polymerase reads the DNA string and makes a messenger RNA copy (mRNA) in a process called transcription. This mRNA copy travels to the ribosome, the protein assembly factory of the cell. The ribosome allows the RNA bases to be read three at a time, and transfer RNA (tRNA) adds the coded-for amino acid to the new protein during the process of translation. When a stop codon is reached, the protein is released, and chaperone proteins help guide it to fold correctly.

Not every part of DNA codes for a gene. Those that do are called exons. Other long stretches of noncoding DNA that

\footnotetext{
a. cwgenna@gmail.com
}

Share this:

were once thought to be genetic junk are actually important control regions. These regions contain the following: promoter regions that increase or decrease the frequency that nearby genes are transcribed; instructions for different kinds of RNA that alter proteins after they are assembled but before they are folded (post-translational modification); and other kinds of RNA that interfere with translation of already transcribed mRNAs or otherwise modify gene function. Transcription and translation have numerous quality control mechanisms built in and DNA can repair itself from certain kinds of damage. If an error persists despite these safeguards, a mutation happens. The three kinds of errors are additions, deletions, and substitutions of bases.

Many mutations are deleterious or bad for the organism. Some are adaptive, or helpful in the current environment. If a base is added into or subtracted from a gene, it causes a frameshift error; this means that the three-base code will not read the same from the error on. That's like adding a single extra letter somewhere in this sentence, and then pushing every other letter or space to the right one place and trying to read it. We can see that the effects may be worse if the frameshift error is early in the gene versus toward the end.

Missense (point) mutations happen when one of the bases is substituted for another, resulting in a wrong amino acid in the protein. Many genes associated with increased cancer risk have single missense mutations. Truncating mutations (inadvertent stop codon insertion) form a very short protein product, or none at all. A protein with a slight change might be able to fit in its receptor, but might not be able to do its expected job once there (di Masi, 2008). Having an inactive protein compete for binding sites with an active protein is like having untrained workers take up work stations in a factory but then just stand there with their arms folded.

\section{Epigenetics}

Cell differentiation allows us to begin as a single fertilized egg, which undergoes many cell divisions and allows the cells to become different kinds of tissues and organs. If the whole genome is a universal appliance construction manual, and we just need to make an oven, we can open to just the oven chapter and keep the rest of the book closed. Now imagine there are two chapters for ovens: one for gas ovens, and one for electric. If we confuse the two sets of instructions, we could wind up with an oven that does not work or that is 
unsafe! To avoid confusing instructions or duplicating some steps, some genes are imprinted, or turned on or off according to the gender of the parent of origin. Imprinting marks are passed to daughter cells during cell division, but they need to be erased and reset during the production of eggs or sperm. Errors of imprinting may adversely affect health and/or development (Robertson, 2005). Early embryonic development is another time when epigenetics are reset, as cells differentiate and primordial tissues develop (Phillips, 2008).

Many epigenetic processes control which genes get read and which get repressed or hidden from transcriptional enzymes. Human DNA is very long and gets packaged into chromosomes by proteins called histones. Histones wind the DNA tightly when the cell is ready to divide, to prevent pieces from getting lost. When the cell is active but not dividing, the parts of the DNA being used are unwound while repressed areas are tightly wound. Chemical changes (modifications) to specific histone amino acids are responsible for winding or unwinding areas of DNA.

DNA methylation is another epigenetic mechanism that works in concert with histone modifications. Control areas in DNA often contain repeats of the base sequence CG, called CpG islands. When these regions are methylated, enzymes that modify histones to wind the chromosome tightly are drawn to these methylated areas and gene expression is repressed. Conversely, $\mathrm{CpG}$ islands with low methylation are associated with histone modifications that unwind the chromosome, so the DNA can be "read" (Zhang, Cooper, \& Brockdorff, 2015). Areas that tend to be highly methylated are repeat DNA segments and transposons, unstable areas that are particularly vulnerable to mutations and chromosome recombination. Housekeeping genes (those that control basic cell life functions) are usually not methylated and generally attract enhancer enzymes that increase their transcription (Liu et al., 2016). Different cell types have characteristic average methylation patterns (Liu et al., 2016), although individual cells may differ from their neighbors even in the same tissue.

Even in nonimprinted areas of DNA, maternal nutrition at conception may affect methylation at $\mathrm{CpG}$ islands. Metastable epialleles are genes that can be turned on or off by differences in methylation soon after conception, producing phenotype differences that are due to epigenetic rather than genetic changes (Anderson, Sant, \& Dolinoy, 2012). Methylation at metastable epialleles are mitotically stable-they are copied along with the DNA during normal cell division, spreading the change to multiple tissues in the early embryo. These epigenetic changes may impact disease susceptibility (Harris, Nagy-Szakal, \& Kellermayer, 2013), including decreased protection from cancer
(Romanelli et al., 2014). Gambian mothers who conceived during the rainy season, when protein food availability was decreased, had infants with increased DNA methylation at metastable epialleles compared to those who conceived during the harvest season. Higher infant DNA methylation was seen when mothers had lower body mass index, homocysteine, cysteine, and vitamin B6 levels (DominguezSalas et al., 2014). Epigenetics is a potential mechanism of metabolic programming of the fetus (Chmurzynska, 2010). Vitamin supplementation during pregnancy needs careful consideration. Either a lack of sufficient nutrients, or supplementation that disrupts the relative proportions the body expects, can have lifelong adverse effects on the fetus' metabolic and cardiovascular health, and cancer risk (Chmurzynska, 2010).

Epigenetic changes can occur over the course of a lifetime in response to environmental exposures, nutrition, and health behaviors and affect the appearance and function of the body. The total epigenetic complement (epigenome) of monozygotic ("identical") twins are more similar during early childhood than later in life, and diverge more in twins with different lifestyles and less time spent together (Fraga et al., 2005).

\section{Epigenetic Effects of Breastfeeding}

A search for epigenetics and breastfeeding on Google Scholar returned almost 12,000 results. Few are reports of basic research. A systematic review of research on differential DNA methylation by breastfeeding exposure (Hartwig, Loret de Mola, Davies, Victora, \& Relton, 2017) identified five human studies. Only one study looked at children who had been fully breastfed. The number of weeks of any breastfeeding had small, nonstatistically significant effects on methylation of the interleukin-4 receptor gene's related $\mathrm{CpG}$ island, which was associated with asthma risk in young women in the United Kingdom (Soto-Ramírez et al., 2013). There were only 200 subjects with full breastfeeding and methylation data.

Dose of full breastfeeding (none, less than 3 months, more than 3 months) was associated with different patterns of whole genome methylation in a case-control study of asthma in 200 children from industrial vs rural areas in the Czech Republic (Rossnerova et al., 2013). No further analysis of this methylation difference was performed.

Leptin (LEP) is a hormone that is important in growth, insulin sensitivity, and appetite control. Obermann-Borst and colleagues examined LEP promoter methylation in toddlers in relation to length of breastfeeding (ObermannBorst et al., 2013). Children who were breastfed at least 1 to 3 months had lower LEP promoter methylation in 
white blood cells, and higher serum levels of leptin, than children who were never breastfed. Children with higher birthweights also had lower LEP methylation. Conversely, foundational studies in epigenetics demonstrated many growth-related gene promoters were hypermethylated in children conceived during the Dutch Hunger Winter famine. A case-control study in women with breast cancer examined early exposures in relation to methylation of cancer associated genes (Tao et al., 2013). This study found that women who were premenopausal, and were never breastfed, were almost three times more likely to have promoter methylation in the p53 gene-a cell-cycle controller and important tumor suppressor (Tao et al., 2013). This suggests epigenetic suppression of protective proteins in those who were never breastfed, providing a potential mechanism for the evidence demonstrating protection against premenopausal breast cancer from breastfeeding, and having been breastfed.

The final study (Simpkin et al., 2016) looked longitudinally at age-related increase in global methylation in blood at birth, and at 7 and 17 years in association with many maternal, pregnancy, and birth-related factors and including being ever or never breastfed. Unsurprisingly with this study design, there were no significant correlations with breastfeeding.

Many other factors influence epigenetics during prenatal development, including the maternal microbiome (Kumar et al., 2014). Kumar and colleagues' pilot study $(n=8)$ showed more methylation in promoters of genes associated with cardiovascular and metabolic risk (inflammation, lipid synthesis, obesity) in women with predominant Firmacutes versus Bacteroidetes/Proteobacteria gut flora. These flora were chosen for comparison because prior research has associated high levels of Firmacutes in the gut with obesity, and obesity with increased inflammation and risk for metabolic syndrome and diabetes, cardiac diseases, and cancer.

Infant stress associated with adverse intrauterine conditions and neonatal intensive care unit care also potentially affect DNA methylation in infancy (Provenzi, Guida, $\&$ Montirosso, 2018). Analysis of newborn cord blood demonstrated that maternal depression during pregnancy is associated with increased methylation of genes controlling growth (Liu et al., 2012). The complex interactions between maternal nutrition (Remely, Stefanska, Lovrecic, Magnet, \& Haslberger, 2015), antibiotic exposure (Lemas et al., 2016), and breastfeeding may prove difficult to tease out.

Breastfeeding may also expose infants to less benign epigenetic effects of mother's environmental exposures or health behaviors. Eight-week-old infants in mothers who drank moderate amounts of alcohol during breastfeeding had increased methylation of an important dopamine receptor (in cheek cells) compared to those who did not drink (Fransquet et al., 2016). The researchers tried to control for drinking during pregnancy by comparing women who drank only postpartum to those who also drank during the third trimester. Methylation analysis both at birth and at 8 weeks would have allowed better discrimination between prenatal and postnatal epigenetic effects of alcohol. The brain is the most relevant tissue to examine dopaminereceptor methylation in, but is obviously invasive to sample in living infants. Cheek cells are derived from the same primordial germ layer as the brain, and similar changes in methylation of cheek-cell dopamine receptors are seen in relapsed adult alcoholics. If further studies confirm that maternal drinking during breastfeeding impacts methylation of important dopamine-system genes in infants, mothers should be supported to not drink.

\section{Conclusion}

Existing studies give tantalizing hints that breastfeeding affects gene methylation in infants in potentially protective ways. Long noncoding RNAs and other RNAs involved in epigenetic mechanisms have been identified in human milk (Munch et al., 2013), but their function is so far unknown. Research on epigenetic effects of breastfeeding is clearly still in its infancy.

\section{References}

Anderson, O. S., Sant, K. E., \& Dolinoy, D. C. (2012). Nutrition and epigenetics: An interplay of dietary methyl donors, one-carbon metabolism and DNA methylation. The Journal of Nutritional Biochemistry, 23(8), 853-859. http://dx.doi.org/10.1016/j.jnutbio. $\underline{2012.03 .003}$

Chmurzynska, A. (2010). Fetal programming: Link between early nutrition, DNA methylation, and complex diseases. Nutrition Reviews, 68(2), 87-98. http://dx.doi.org/10.1111/i.1753-4887. 2009.00265.x

di Masi, A. (2008). May a missense mutation be more deleterious than a truncating mutation? IUBMB Life, 60(1), 79-81. http:// dx.doi.org/10.1002/iub.2

Dominguez-Salas, P., Moore, S. E., Baker, M. S., Bergen, A. W., Cox, S. E., Dyer, R. A., . . Hennig, B. J. (2014). Maternal nutrition at conception modulates DNA methylation of human metastable epialleles. Nature Communications, 5, 3746. http://dx.doi.org/10. $1038 /$ ncomms 4746

Fraga, M. F., Ballestar, E., Paz, M. F., Ropero, S., Setien, F., Ballestar, M. L., ... Esteller, M. (2005). Epigenetic differences arise during the lifetime of monozygotic twins. Proceedings of the National Academy of Sciences of the United States of America, 102(30), 1060410609. http://dx.doi.org/10.1073/pnas.0500398102

Fransquet, P. D., Hutchinson, D., Olsson, C. A., Wilson, J., Allsop, S., Najman, J., . . Triple B Research Consortium. (2016). Perinatal 
maternal alcohol consumption and methylation of the dopamine receptor DRD4 in the offspring: The triple B study. Environmental Epigenetics, 2(4), dvw023. http://dx.doi.org/10.1093/eep/dvw023

Harris, R. A., Nagy-Szakal, D., \& Kellermayer, R. (2013). Human metastable epiallele candidates link to common disorders. Epigenetics, 8(2), 157-163. http://dx.doi.org/10.4161/epi.23438

Hartwig, F. P., Loret de Mola, C., Davies, N. M., Victora, C. G., \& Relton, C. L. (2017). Breastfeeding effects on DNA methylation in the offspring: A systematic literature review. PLoS One, 12(3), e0173070. http://dx.doi.org/10.1371/journal.pone.0173070

Kumar, H., Lund, R., Laiho, A., Lundelin, K., Ley, R. E., Isolauri, E., \& Salminen, S. (2014). Gut microbiota as an epigenetic regulator: Pilot study based on whole-genome methylation analysis. mBio, 5(6), e02113-14 http://dx.doi.org/10.1128/mBio.02113-14

Lemas, D. J., Yee, S., Cacho, N., Miller, D., Cardel, M., Gurka, M., . .. Shenkman, E. (2016). Exploring the contribution of maternal antibiotics and breastfeeding to development of the infant microbiome and pediatric obesity. Seminars in Fetal and Neonatal Medicine, 21(6), 406-409. http://dx.doi.org/10.1016/j.siny.2016. $\underline{04.013}$

Liu, H., Liu, X., Zhang, S., Lv, J., Li, S., Shang, S., .. Zhang, Y. (2016). Systematic identification and annotation of human methylation marks based on bisulfite sequencing methylomes reveals distinct roles of cell type-specific hypomethylation in the regulation of cell identity genes. Nucleic Acids Research, 44(1), 75-94. http://dx.doi. org/10.1093/nar/gkv1332

Liu, Y., Murphy, S. K., Murtha, A. P., Fuemmeler, B. F., Schildkraut, J., Huang, Z., . . . Hoyo, C. (2012). Depression in pregnancy, infant birth weight and DNA methylation of imprint regulatory elements. Epigenetics, 7(7), 735-746. http://dx.doi.org/10.4161/ epi.20734

Munch, E. M., Harris, R. A., Mohammad, M., Benham, A. L., Pejerrey, S. M., Showalter, L., . . Aagaard, K. (2013). Transcriptome profiling of microRNA by Next-Gen deep sequencing reveals known and novel miRNA species in the lipid fraction of human breast milk. PLoS One, 8(2), e50564. http://dx. doi.org/10.1371/journal.pone.0050564

Obermann-Borst, S. A., Eilers, P. H., Tobi, E. W., de Jong, F. H., Slagboom, P. E., Heijmans, B. T., \& Steegers-Theunissen, R. P. (2013). Duration of breastfeeding and gender are associated with methylation of the LEPTIN gene in very young children. Pediatric Research, 74(3), 344-349. http://dx.doi.org/10.1038/pr.2013.95

Phillips, T. (2008). The role of methylation in gene expression. Nature Education, 1(1), 116.
Provenzi, L., Guida, E., \& Montirosso, R. (2018). Preterm behavioral epigenetics: A systematic review. Neuroscience $\mathcal{E}$ Biobehavioral Reviews, 84(1), 262-271. http://dx.doi.org/10. 1016/j.neubiorev.2017.08.020

Remely, M., Stefanska, B., Lovrecic, L., Magnet, U., \& Haslberger, A. G. (2015). Nutriepigenomics: The role of nutrition in epigenetic control of human diseases. Current Opinion in Clinical Nutrition $\mathcal{E}$ Metabolic Care, 18(4), 328-333. http://dx.doi.org/10.1097/MCO. $\underline{0000000000000180}$

Robertson, K. D. (2005). DNA methylation and human disease. Nature Reviews Genetics, 6(8), 597-610. http://dx.doi.org/10.1038/ $\underline{\operatorname{nrg} 1655}$

Romanelli, V., Nakabayashi, K., Vizoso, M., Moran, S., IglesiasPlatas, I., Sugahara, N., . . Monk, D. (2014). Variable maternal methylation overlapping the nc886/vtRNA2-1 locus is locked between hypermethylated repeats and is frequently altered in cancer. Epigenetics, 9(5), 783-790. http://dx.doi.org/10.4161/epi. $\underline{28323}$

Rossnerova, A., Tulupova, E., Tabashidze, N., Schmuczerova, J., Dostal, M., Rossner, P., . . Sram, R. J. (2013). Factors affecting the $27 \mathrm{~K}$ DNA methylation pattern in asthmatic and healthy children from locations with various environments. Mutation Research/Fundamental and Molecular Mechanisms of Mutagenesis, 741-742, 18-26. http://dx.doi.org/10.1016/j.mrfmmm.2013.02. $\underline{003}$

Simpkin, A. J., Hemani, G., Suderman, M., Gaunt, T. R., Lyttleton, O., Mcardle, W. L., ... Smith, G. D. (2016). Prenatal and early life influences on epigenetic age in children: A study of motheroffspring pairs from two cohort studies. Human Molecular Genetics, 25(1), 191-201. http://dx.doi.org/10.1093/hmg/ddv456

Soto-Ramírez, N., Arshad, S. H., Holloway, J. W., Zhang, H., Schauberger, E., Ewart, S., . . Karmaus, W. (2013). The interaction of genetic variants and DNA methylation of the interleukin-4 receptor gene increase the risk of asthma at age 18 years. Clinical Epigenetics, 5(1), 1), 1. http://dx.doi.org/10.1186/ $\underline{1868-7083-5-1}$

Tao, M. H., Marian, C., Shields, P. G., Potischman, N., Nie, J., Krishnan, S. S., . . Freudenheim, J. L. (2013). Exposures in early life: Associations with DNA promoter methylation in breast tumors. Journal of Developmental Origins of Health and Disease, 4(2), 182-190. http://dx.doi.org/10.1017/S2040174412000694

Zhang, T., Cooper, S., \& Brockdorff, N. (2015). The interplay of histone modifications-writers that read. EMBO Reports, 16(11), 1467-1481. http://dx.doi.org/10.15252/embr.201540945

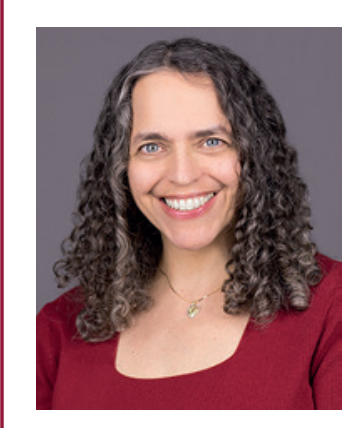

Catherine Watson Genna, BS, IBCLC, RLC, has been an IBCLC in private practice in New York City since 1992. She is the author of Supporting Sucking Skills in Breastfeeding Infants (Jones and Bartlett Learning, 2008, 2013, 2017) and Selecting and Using Breastfeeding Tools (Hale Publishing, 2009; Praeclarus, 2016) and served as a founding Associate Editor of the USLCA journal, Clinical Lactation, for 7 years. 